\title{
Construct Validity and Measurement Invariance of the Research Skill Inventory
}

\author{
Sanit Srikoon ${ }^{1}$ \\ Tassanee Bunterm ${ }^{1^{*}}$ \\ Teerachai Nethanomsak ${ }^{1}$ \\ Tang Keow Ngang ${ }^{2}$ \\ ${ }^{1}$ Faculty of Education, Khon Kaen University, Khon Kaen 40002, Thailand \\ *Corresponding Author Email: tassaneebun@gmail.com \\ 2International College, Khon Kaen University, Khon Kaen 40002, Thailand
}

\section{Doi:10.5901/mjss.2016.v7n3p366}

\begin{abstract}
This paper aimed to validate the developmental process of instrument to create an instructional design model. Therefore this study was designed to test the construct validity of research skill inventory and examine the measurement invariance of research skills across gender and grade. The effect sampling size was 1969 samples. However the actual samples participated was 1712, given a response rate as 86.94 percent. Data was analyzed to test construct validity and multi-group confirmatory factor analysis across gender and grades. Confirmatory factor analysis supports the six models consists of male, female, Grade 7, Grade 8, Grade 9, and total CFA models. MGCFA was comprised of measurement invariance and structural invariance. Findings indicated that measurement invariance had been proved to support the four models as non-invariance namely gender, Grade 7-Grade 8, Grade 8-Grade 9, and Grade 7-Grade 9 models. Structural invariance testing had been proved to support the three invariance models except Grade 8-Grade 9 model.
\end{abstract}

Keywords: Research skills, construct validity, measurement invariance

\section{Introduction}

A growing body of literature suggests research skills (RSs) are related to student's learning, attitude, research competency, critical thinking, and academic achievement (Wannapiroon, 2014; Srikoon, Bunterm, Samranjai \& Wattanathorn, 2014; Zehra, Hassaan \& Mushtaq, 2015; Stappenbelt, 2013). Consequently RSs are recognized as important contributors to children behavior and have become the focus of learning process or so called as 'student as researcher' (Elizabeth \& Grant, 2013; Leat \& Reid, 2012; Wilkin, 2014).

\section{Problem Statement}

Despite its wide-spread mention, little research has investigated the RSs, and only a few studies have defined the factor structure of RSs. For example, Stokking*, Schaaf, Jaspers, and Erkens (2004) refer RSs as teachers' assessment for students' RSs were complex skills but were undefined them. Although Kiley, Moyes and Clayton (2009), and Czarneski (2013) reported RSs in their research but they were not clearly defined it, too. To date, only a few studies have referred the factor structure of RSs (Meerah et al., 2012; Willison, 2007).

\section{Significant of the Study}

Construct validity (CV) is designed to measure the theoretical latent construct of variables in order to assess the quality of measures of a behavioral model (Hair, 2006). In addition, confirmatory factor analysis (CFA) is utilized when research has some knowledge of the underlying latent variable structure (Bryne, 2012). The combination of CFA results and CV tests can obtain a better understanding of the quality of these measures (Hair, 2006). Therefore, many researchers used CFA to confirm CV, like Canivez and Sproul (2005), Canivez, Neitzel, and Martin (2005), Martin and Marsh (2008), and Weis and Smenner (2007). In conclusion, CFA is the approximately method to test how well the measured variables represent 
the construct.

One of the social science variables for managing learning in classrooms were gender and grade (Lowe, 2014). To date, gender and grade are considered for learning measurement condition (Bas \& Yurdabakan, 2012; Harrell-Williams, Sorto, Pierce, Lesser, \& Murphy, 2014; Lowe, 2014; Siegling, Furnham, \& Petrides, 2015).

\section{Conceptual Framework}

Research skill inventory is the self-assessment inventory for diagnosing face validity. It consists of 35 items of seven factors. These seven RSs are Research Questioning Skill (ROS), Research Literacy Skill (RLS), Research Design Skill (RDS), Research Collecting Data Skill (RCDS), Research Organizing Data Skill (RODS), Research Conclusion Skill (RCS), and Research Result Presenting Skill (RRPS). Figure 1 shows the conclusion of the seven research skills. Each statement of research instrument is using a 5-point Likert scale namely 1 represents 'Does not perform', 2 represents 'Does not really perform', 3 represents 'Neutral, not sure', 4 represents 'Perform somewhat', and 5 represents 'Absolutely perform'

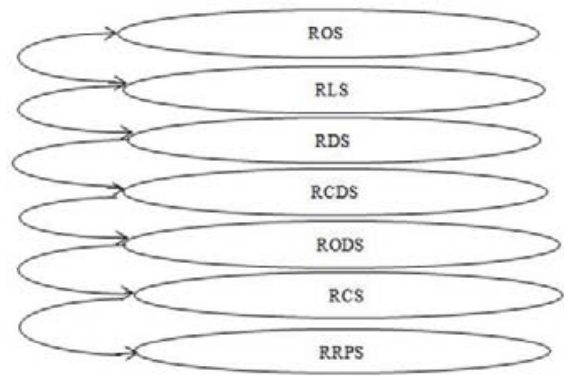

Figure 1: Conceptual framework

\section{Aim of the Study}

RSs should be precisely measured to examine the nature of construct and to make valid explanations for each individual's social activity. This study aimed to validate the RSs and further address measurement invariance across gender and grade. The specific purposes of this study are as follow:

1. To test the CV of the RSs with the consistency idea in each gender and grade.

2. To examine the measurement invariance of RSs across gender and grade.

\section{Research Methodology}

\subsection{Sample of the Study}

A total of $1969(N)$ samples were drawn from Grade $7\left(n_{1}=648\right)$, Grade $8\left(n_{2}=660\right)$, and Grade $9\left(n_{3}=661\right)$. Owing to incomplete response, a total of 257 cases have been excluded for further analyzing namely 2 from Grade 7, 83 from Grade 8, and 172 from Grade 9, given the actual samples as 1712. As a result, there were 646 (37.73\%) Grade 7 cases consisted of 415 males (24.24\%) and 231 females (13.49\%), 577 (33.71\%) Grade 8 cases encompassed 206 males (12.04\%) and 371 females (21.67\%), and 489 (28.56\%) Grade 9 cases included 204 males (11.92\%) and 285 females (16.64\%).

\subsection{Method of the Study}

The rapid growing of advanced methodology would be provided the ability of researchers to analyze the CV across group so called 'multiple group invariance confirmatory factor analysis' (MGCFA). MGCFA is used to compare latent variable means, variances, and co-variances across groups while holding measurement parameter invariant (Asparouhov \& Muthen, 2014; Wang \& Wang, 2012). In other word, MGCFA consists of two different kinds of invariance namely 
measurement invariance and structural invariance.

Measurement invariance is tested first and followed by structural invariance. Four different level of measurement invariance are measurement configurable invariance, weak measurement invariance, strong measurement invariance, and strict measurement invariance. Meanwhile structural invariance consists of three levels namely invariance of factor variance, invariance of factor covariance, and factor mean invariance.

MGCFA can give the elaborately results about metric invariance, scalar invariance, and invariance of structural parameter across group (Wang \& Wang, 2012; Dimitrov, 2010). There are two reasons of using MGCFA namely to ensure underlying construct has the same theoretical structure and psychological meaning across the groups (Bryne, 2008) as well as to examine the equivalence of factorial validity across gender and grade.

\subsection{Data Analysis}

Other than descriptive statistics, CFA Mplus program version 6 is utilized to test the hypothesized of seventh-factor model consisted of 35 items in all samples, for each gender and grade. Covariance structures are fitted with the maximum likelihood method. The model fitted indicator is evaluated by means of several fit indices. Universally, the model is considered acceptable when probability value ( $p$-value) $>0.05$, value of ratio between chi-square statistic and degree of freedom ( $X^{2 / d f)}$ in 2:1 (Hair, 2006), the Comparative Fit Index (CFI) $\geq 0.90$ and good when $\geq 0.95$ (Bentler, 1992; Hu \& Bentler, 1999) and Tucker-Lewis coefficient (TLI) $\geq 0.95$ is good fit (Sharma, Mukherjee, Kumar, \& Dillon, 2005). Moreover, Standardized Root Means Square Residual (SRMR) should not exceed 0.08 for a good fit (Hu \& Bentler, 1999). Furthermore, Root Means Square Error of Approximation (RMSEA) value $\leq 0.06$ are considered indicative of a good fit, $\leq 0.08$ of fair fit, between 0.08 and 0.01 of mediocre fit and $>0.01$ of poor fit (Hu \& Bentler, 1999; MacCallum, Browne, \& Sugawara, 1996).

Analyzing of MGCFA were based on the Satorra-Bentler scaled chi-square statistics and usual the Maximum Likelihood (MLx2statistic) because it serves a correction of chi-square when distributional assumption are violated. In testing model can evaluate the goodness-of-fit of model by multiple criteria including CFI, RMSEA, 90 percent confidence interval, and SRMR (Byrne, 2008). CFI values in the range of 0.92 to 0.94 may also be considered as reasonable indicators of good model fit (Marsh, Hau \& Wen, 2004). RMSEA values less than 0.05 indicates good fit, and value as high as 0.08 represent reasonable errors of approximation in the popular (M.W. Browne, 1992). For completeness, Bryne (2008) guides to report the 90 percent confidence interval provide for RMSEA (Steiger, 1990). Lastly, SRMR values range from 0.00 to 1.00, with a value less than 0.08 being indicative of a well-fitting model (Hu \& Bentler, 1999).

\section{Research Findings}

Findings are presented in three sections namely descriptive findings, CV, and measurement invariance of gender and grade.

\subsection{Descriptive Findings}

Descriptive statistics like mean value $(\bar{x})$, standard deviation (S.D.), skewness (sk) and kurtosis (ku) are used to analyze the 35 items of the RSs. All items in the research skill inventory were distributed normally according to gender, grade and the total when compared with criteria because skewness values between 2 and -2 ad kurtosis values between 7 and -7 have been estimated as normal as presented in Table 1.

\subsection{Construct Validity}

CFA was used to evaluate the goodness of fit. Model fit was assessed using $X^{2}, X^{2} / \mathrm{df}, \mathrm{CFI}, \mathrm{TLI}, \mathrm{SRMR}$ and RMSEA. Fit indices of all CFA models, including total, male, female, grade 7, grade 8, and grade 9 models are presented in Table 2. Results showed that all indicators indicated that there was a goodness of fit between the empirical data and the hypothetical measurement model for all models. In other word, all the $p$-value in CFA models are not significant (.053$.067), X^{2} /$ df values are fallen in 2:1 (1.095-1.102), all CFA $\geq 0.95$ indicate the good fit (.988-.997). Similarity all the TLIs $\geq$ 0.95 are good fit (.987-.996). Moreover all the RMSEAs are $\leq 0.06$ are considered indicative of a good fit (.008-.014). Finally all the SRMRs $\leq 0.08$ are accepted for a good fit (.019-.033) too.

Individual parameter estimated for all the CFA models were also examined. Table 3 lists the standardized factor 
loadings each latent variables of any CFA models. Meanwhile Table 4 shows the factor correlations between latent variables and factor loading of items in each CFA models were all positive and statistically significant.

Table 1. Mean, standard deviation, skewness, and kurtosis

\begin{tabular}{|c|c|c|c|c|c|c|c|c|c|c|c|c|c|c|c|c|c|c|c|c|c|c|c|c|}
\hline \multirow{3}{*}{ Items } & \multicolumn{8}{|c|}{ gender } & \multicolumn{12}{|c|}{ Grade } & \multirow{2}{*}{\multicolumn{4}{|c|}{ Total $(n=1712)$}} \\
\hline & \multicolumn{4}{|c|}{ Male $(n=641)$} & \multicolumn{4}{|c|}{ Female $(n=1071)$} & \multicolumn{4}{|c|}{ Grade7(n=646) } & \multicolumn{4}{|c|}{ Grade $8(n=577)$} & \multicolumn{4}{|c|}{ Grade $9(n=489)$} & & & & \\
\hline & $\bar{x}$ & S.D. & sk & $\mathrm{ku}$ & $\bar{x}$ & S.D. & sk & $\mathrm{ku}$ & $\bar{x}$ & S.D. & sk & $\mathrm{ku}$ & $\bar{x}$ & S.D & sk & $\mathrm{Ku}$ & $\bar{x}$ & S.D & sk & $\mathrm{ku}$ & $\bar{x}$ & S.D. & sk & $\mathrm{ku}$ \\
\hline em 1 & 3.73 & .84 & .02 & .48 & 3.77 & .82 & .00 & -.62 & 3.64 & 84 & 20 & .55 & 3.90 & .80 & \begin{tabular}{|c|}
-.08 \\
\end{tabular} & $\mid-80$ & 3.74 & 81 & .13 & .20 & 1.6 & 83 & .01 & .57 \\
\hline 2 & .59 & .89 & 02 & 37 & 3.70 & 88 & 17 & 29 & 3.42 & 87 & .09 & .09 & 79 & .88 & 60 & 41 & 3.82 & .83 & 21 & 52 & .00 & 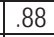 & 11 & -.34 \\
\hline 3 & 64 & .85 & 0 & & & 82 & & & & 82 & 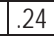 & & & 81 & & & & & & & & & & \\
\hline 4 & 55 & .85 & 05 & 8 & 50 & 83 & 16 & 18 & 3.45 & 84 & 23 & & 56 & .82 & 00 & -.06 & & 85 & & & 50 & & & \\
\hline 5 & 23 & .88 & .15 & $\underline{0}$ & 3.21 & 82 & 38 & .28 & 3.15 & 82 & 34 & 52 & 30 & .82 & .33 & .02 & 3.21 & 87 & 19 & 07 & 3.22 & 84 & 28 & 16 \\
\hline em 6 & 17 & .84 & .06 & 29 & 3.16 & .85 & .09 & .22 & 3.17 & .81 & 22 & .67 & 3.20 & .87 & \begin{tabular}{|l|}
.01 \\
\end{tabular} & .05 & 3.11 & .86 & .00 & .00 & 3.16 & 85 & 08 & .24 \\
\hline Item 7 & 3.39 & .84 & .01 & .01 & 3.37 & .81 & .12 & .08 & 32 & 77 & 23 & .50 & 44 & .85 & \begin{tabular}{|l|}
.02 \\
\end{tabular} & .29 & 3.36 & .85 & .03 & .05 & 3.37 & 32 & 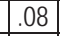 & 0 \\
\hline em 8 & 3.41 & \begin{tabular}{|l}
.84 \\
\end{tabular} & .03 & 19 & 3.37 & .77 & .11 & .10 & 3.36 & .79 & .20 & .44 & 47 & .77 & \begin{tabular}{|l|}
.10 \\
\end{tabular} & -.01 & 3.33 & .82 & -.03 & .01 & 3.39 & 79 & 88 & .16 \\
\hline$m 9$ & 34 & .82 & .15 & 08 & 3.35 & .78 & 14 & .04 & 3.33 & .78 & .20 & 20 & 38 & .80 & \begin{tabular}{|l|}
.07 \\
\end{tabular} & .04 & 3.32 & .82 & 17 & 18 & 3.34 & 80 & & 0 \\
\hline$m 10$ & 3.30 & .87 & .10 & 3 & 3.35 & .83 & .20 & .08 & 3.29 & 85 & 20 & 5 & 3.38 & .84 & 15 & -.13 & 10 & .86 & .11 & .09 & 3.33 & & 15 & .0 \\
\hline $\mathrm{m} 11$ & 3.40 & .83 & 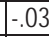 & 1 & 3.44 & .83 & .05 & .05 & 36 & 81 & .25 & 4 & 3.51 & .83 & -.23 & .25 & 3.41 & .84 & .03 & -.08 & 3.4 & & 02 & 05 \\
\hline $\mathrm{m} 12$ & 3.38 & .87 & .13 & .30 & 3.39 & .84 & .07 & .03 & 3.35 & 83 & 32 & .02 & 3.44 & \begin{tabular}{|l}
.87 \\
\end{tabular} & .07 & -.20 & 3.37 & .84 & .00 & 12 & 3.5 & & & -.1 \\
\hline $\mathrm{m} 13$ & 3.33 & .92 & .09 & 28 & \begin{tabular}{|l|l}
3.40 \\
\end{tabular} & .90 & .13 & 51 & 3.39 & 87 & 25 & .21 & 3.47 & 92 & 02 & 61 & 207 & 92 & .11 & 39 & 4 & 5 & \pm \pm & \\
\hline$m 14$ & 3.24 & .86 & 22 & 3 & 3.22 & 85 & 11 & .07 & 3.23 & .84 & 18 & 5 & 3.28 & .84 & \begin{tabular}{|l|l|} 
\\
\end{tabular} & -.15 & 3.18 & 88 & .14 & -.08 & 3.23 & 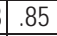 & & 05 \\
\hline 5 & 3.35 & .86 & .06 & 05 & 3.27 & .86 & .09 & .06 & 3.27 & 84 & 13 & 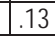 & 31 & .88 & .03 & -.04 & 3.33 & 87 & .14 & 30 & 3.30 & & & -.0 \\
\hline $\mathrm{n} 16$ & 3.39 & .91 & -.05 & 2 & 3.37 & .86 & .06 & -.15 & 3.33 & .83 & .23 & 0 & 3.42 & .89 & .10 & -.13 & 3.39 & 91 & -.09 & .22 & 3 & & JL & .1 \\
\hline n 17 & 3.37 & .89 & .06 & 24 & +3.33 & .81 & .14 & .10 & 3.34 & 87 & 19 & 5 & 3.36 & .92 & \begin{tabular}{|l|}
.05 \\
\end{tabular} & -.39 & 3. & 4 & 08 & 12 & 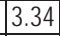 & 00 & 11 & -.1 \\
\hline $\mathrm{m} 18$ & 3.44 & .85 & .07 & .02 & 3.50 & .82 & 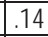 & .36 & 3.39 & 81 & .29 & 8 & 3.60 & .84 & \begin{tabular}{|l|}
.10 \\
\end{tabular} & -.57 & 3.46 & .83 & -.14 & .06 & $3.4 \varepsilon$ & 83 & 11 & 2 \\
\hline m 19 & 3.46 & .85 & .11 & .36 & 3.53 & .86 & -.04 & .18 & 3.41 & 84 & .20 & .08 & 3.61 & .84 & .09 & -.33 & 3.50 & 88 & -.09 & 44 & 3.50 & & 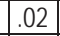 & .2 \\
\hline 120 & 3.39 & .89 & .07 & 20 & 3.43 & .87 & .06 & .25 & .39 & 87 & 23 & 2 & 3.47 & .85 & -.01 & \begin{tabular}{|l} 
\\
\end{tabular} & 3.37 & 92 & -.04 & \begin{tabular}{|l|l}
4.29 \\
\end{tabular} & 3.41 & 88 & 10 & -.23 \\
\hline 21 & 3.40 & .92 & -.04 & & 3.58 & 89 & -05 & .44 & 3.42 & 85 & .16 & & & .93 & .16 & -.43 & 3.51 & .92 & -.22 & -.28 & 3.51 & & & .24 \\
\hline $\mathrm{m} 22$ & 3.42 & .89 &. .10 & & 53.56 & .86 & 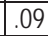 & -.47 & 3.45 & 82 & .29 & $\perp$ & 40 & .92 & .19 & -.48 & 3.49 & 89 & .11 & .15 & 3.5 & & 01 & .3 \\
\hline $\mathrm{m} 23$ & 3.43 & .88 & -.09 & .07 & 3.5 & 86 & .06 & 38 & 3 & .83 & .18 & \pm & t & .90 & .09 & -.45 & 3.47 & 88 & -.14 & \begin{tabular}{|l|l}
4.20 \\
\end{tabular} & 3.4 & 87 & 00 & -.10 \\
\hline $\mathrm{m} 24$ & 3.39 & .90 & -.03 & 18 & 3.39 & .87 & .11 & .37 & 3 & 86 & .07 & 6 & 3.45 & .89 & .09 & -.65 & 3.38 & 90 & -.01 & -.29 & 3.3 & 88 & 05 & .2 \\
\hline $\mathrm{m} 25$ & 3.37 & \begin{tabular}{|l}
.87 \\
\end{tabular} & .11 & .04 & 3.34 & .90 & .04 & \begin{tabular}{|l}
-14 \\
\end{tabular} & 3.29 & .87 & -.02 & .35 & 3.42 & \begin{tabular}{|l}
.88 \\
\end{tabular} & \begin{tabular}{|l|}
.12 \\
\end{tabular} & \begin{tabular}{|l}
-.32 \\
\end{tabular} & 3.34 & .92 & .09 & -.43 & 3.35 & g & 06 & -.1 \\
\hline $\mathrm{m} 26$ & 3.43 & \begin{tabular}{|l}
.87 \\
\end{tabular} & -.09 & .03 & 3.47 & .86 & .19 & -.30 & 3.37 & .86 & 26 & .15 & 3.60 & .85 & -.03 & \begin{tabular}{l|l}
3.32 \\
\end{tabular} & 3.41 & 1.86 & .00 & -.22 & 3.46 & 86 & .08 & -.16 \\
\hline $\mathrm{m} 27$ & 3.43 & .86 & -.02 & -.27 & 3.46 & .86 & 1 & \begin{tabular}{|l}
-.06 \\
\end{tabular} & 3.38 & 30 & .06 & 38 & 3.55 & .91 & .14 & -.27 & 3.42 & .87 & .02 & .44 & 3.45 & .86 & 1 & -.1 \\
\hline$m<8$ & 3.41 & .87 & .08 & 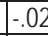 & 3.44 & 86 & 2 & .12 & 3.42 & 83 & 11 & .25 & 3.53 & .88 & 03 & -.40 & 3.33 & 88 & -.05 & 5 & 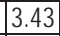 & & & $\Omega$ \\
\hline 29 & 3.47 & .89 & \begin{tabular}{|l}
-01 \\
\end{tabular} & & 33.47 & 86 & 2 & 30 & 3.42 & .86 & .12 & .00 & 3.56 & 87 & \begin{tabular}{|l|}
.01 \\
\end{tabular} & .40 & 3.42 & 88 & .06 & 27 & 3.47 & 87 & .07 & 2 \\
\hline tem 30 & 3.43 & .84 & .12 & .09 & 3.46 & .86 & 9 & .31 & 3.41 & 80 & .44 & .11 & 3.55 & .90 & -12 & .22 & 3.39 & \begin{tabular}{|l|l|}
9.84 \\
\end{tabular} & .18 & .20 & 3.45 & 85 & 17 & 23 \\
\hline em 31 & 3.52 & .92 & -.02 & 40 & 3.70 & .89 & .20 & -.39 & 3.60 & 87 & -.03 & .09 & 3.77 & .93 & \begin{tabular}{|l|}
-33 \\
\end{tabular} & -.51 & 3.53 & .91 & -.06 & \begin{tabular}{|l|l|} 
& -.49 \\
\end{tabular} & 3.64 & 1 & 14 & -.4 \\
\hline Item 32 & 3.39 & .94 & \begin{tabular}{|l|}
-.04 \\
\end{tabular} & -.40 & 3.50 & .90 & .00 & -.36 & 3.40 & 90 & .11 & -.13 & 3.62 & .90 & -18 & -.41 & 3.34 & \begin{tabular}{l|l}
4.93 \\
\end{tabular} & .00 & -.46 & 3.46 & .92 & .02 & -.3 \\
\hline tem 33 & 3.48 & .86 & .13 & -.33 & 3.55 & .86 & .02 & -.26 & 3.47 & .83 & .13 & .09 & 3.60 & .88 & -.13 & -.38 & 3.49 & $\begin{array}{l}9.86 \\
\end{array}$ & .17 & -.53 & 3.52 & 86 & .06 & -.30 \\
\hline $\mathrm{m} 34$ & 3.41 & .85 & .16 & .21 & 3.46 & .83 & .13 & -.16 & 3.38 & .79 & 29 & .30 & 3.54 & .84 & \begin{tabular}{|l|}
.05 \\
\end{tabular} & -.35 & 3.41 & 89 & .06 & .43 & 3.44 & 84 & .14 & -.1 \\
\hline $\mathrm{em} 3$ & 3.45 & .93 & {$[-.03$} & 23 & 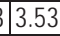 & .86 & .20 & 0 & 45 & .00 & 25 & & & .91 & -.10 & 19 & 47 & . & & & . & .89 & .09 & ]$^{-.34}$ \\
\hline
\end{tabular}

Note: $\mathrm{SE}_{\text {sk(malel) }}=0.097, \mathrm{SE}_{\text {ku(male })}=0.193, \mathrm{SE}_{\mathrm{sk}(\text { (emalel) })}=0.075, \mathrm{SE}_{\text {ku(female })}=0.149, \mathrm{SE} \mathrm{E}_{\text {sk(total) }}=0.059, \mathrm{SE}_{\text {ku(total) }}=0.118$,

$S E_{\text {sk(grade 7) }}=0.096, S E_{k u(\text { grade } 7)}=0.192, S E_{\text {sk(grade 8) }}=0.102, S E_{k u(\text { grade } 8)}=0.203, S E_{s k(\text { grade } 9)}=0.110, S E_{k u(g r a d e ~ 9)}=0.220$

Table 2. Goodness of fit test of alternative models

\begin{tabular}{|l|c|c|c|c|c|c|c|c|}
\hline Group & $x^{2}$ & $\mathrm{df}$ & $\mathrm{p}$-value & $x^{2} / d f$ & $\mathrm{CFI}$ & TLI & RMSEA & SRMR \\
\hline Total & 543.058 & 495 & .067 & 1.097 & .997 & .996 & .008 & .019 \\
\hline Male & 563.071 & 514 & .066 & 1.095 & .989 & .988 & .012 & .031 \\
\hline Female & 548.330 & 499 & .063 & 1.099 & .996 & .995 & .010 & .021 \\
\hline Grade 7 & 575.115 & 522 & .053 & 1.102 & .992 & .990 & .013 & .028 \\
\hline Grade 8 & 559.747 & 511 & .067 & 1.095 & .990 & .988 & .013 & .032 \\
\hline Grade 9 & 567.267 & 515 & .055 & 1.101 & .988 & .987 & .014 & .033 \\
\hline
\end{tabular}




\subsection{Measurement Invariance across Gender and Grade}

Mean least square $(\mathrm{ML})$ was used to test the measurement invariance. Factorial invariance across gender or grade was presented in the measurement invariance and structural invariance. Additionally, measurement invariance consists of testing configural invariance, weak measurement invariance, and strict measurement invariance. Weak measurement invariance is defined as invariance of factor loading across group. Factor loading invariance is also called metric invariance. Strong measurement invariance is defined as invariance of both factor loading and item intercept across group. Strict measurement invariance requires metric invariance, scalar invariance, and error variance invariance across group. Structural invariance across gender consists of the invariance of factor invariance, the invariance of factor covariance, and the factor mean invariance. Testing invariance of factor invariance implies that the factors or latent constructs have the same distribution dispersion across group. Testing covariance represents the association between the two factors when factor invariance holds. Testing mean variance is comparing the factor mean across group.

\subsubsection{Measurement Invariance across Gender}

The model fit similarity for males and females as such $X^{2}(\mathrm{df}=535)=1058.210, p<0.001 ; \mathrm{CFI}=.916$; TLI=.906; SRMR=.038, RMSEA=.039; RMSEA 90\% C.I. = [.036,.043] for males; and $X^{2}(\mathrm{df}=536)=1413.328, p<0.001 ; \mathrm{CFI}=.942$; $\mathrm{TLI}=.935$; SRMR= .030, RMSEA $=.039$; RMSEA $90 \%$ C.I. $=[.037, .042]$ for females. The males and females baseline models for analyzing configural model fit the data well: $X^{2}(d f=1071)=2471.538, p<0.001 ; C F I=.934 ; T L I=.927$; SRMR= .033, RMSEA $=.039 ;$ RMSEA 90\% C.I. $=[.037, .041]$ (Table 5).

Weak measurement indicates of testing metric invariance is $\Delta x^{2}(\mathrm{df}=28)=26.538, p=0.543$ which is not statistically significant. Since one of the factor loading of each factor or 'marker items', including item 1, item 6 , item 11 , item 16, item 21, item 26, and item 31, are not tested in the likelihood ratio (LR) test so they must be tested invariance of the marker items factor loading. The difference in model $x^{2}$ statistics between model with and without equality restriction; $\Delta x^{2}(\mathrm{df}=7)=6.128, p=0.523$ which is not statistically significant, indicating that the factor loading of maker items are invariance across gender.

Strong measurement with LR test of scalar invariance shows as such $\Delta x^{2}(\mathrm{df}=63)=114.571, p=0.000$ which is statistically significant but $\Delta \mathrm{CFI}=0.002$ is lesser than 0.01 . Thus, both factor loading and item intercept of the RSI are non-invariance across gender. Strict measurement with LR test of two nested models of error invariance are $\Delta x^{2}$ ( $\mathrm{df}=98$ ) $=395.44, p=0.000$ of no equality restriction on item invariance which are statistically significant. The corresponding $\Delta x^{2}$ $(\mathrm{df}=35)=280.869, p=0.000$ of equality restriction on item invariance which are statistically significant. Thus, results indicate that item invariances are non-invariant across groups.

Table 3. Parameter estimates of standardized model results

\begin{tabular}{|c|c|c|c|c|c|c|c|c|c|c|c|c|}
\hline \multirow{2}{*}{ Items } & \multicolumn{2}{|l|}{ Male } & \multicolumn{2}{|l|}{ Female } & \multicolumn{2}{|l|}{ Grade 7} & \multicolumn{2}{|l|}{ Grade 8} & \multicolumn{2}{|l|}{ Grade 9} & \multicolumn{2}{|l|}{ Total } \\
\hline & $\beta(\mathrm{SE})$ & $\mathrm{R}^{2}$ & $\overline{\beta(S E)}$ & $\mathrm{R}^{2}$ & $\overline{\beta(S E)}$ & $\mathrm{R}^{2}$ & $\beta(\mathrm{SE})$ & $\mathrm{R}^{2}$ & $\beta(\mathrm{SE})$ & $\mathrm{R}^{2}$ & $\beta(\mathrm{SE})$ & $\mathrm{R}^{2}$ \\
\hline \multicolumn{13}{|l|}{ RQS } \\
\hline 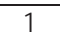 & & 39 & 30)] & .214 & & 270 & & .253 & & 28 & 24) & \\
\hline 2 & & 73 & & 262 & & 267 & (1) & .193 & 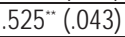 & 10 & 21 & 13 \\
\hline 3 & * $(.034)$ & .348 & $5^{\text {t* }}(.028)$ & 378 & $1^{\text {** }}(.036)$ & .410 & $88^{\text {t* }}(.040)$ & .228 & $3^{*+*}(.037)$ & 317 & $22^{* *}(.022)$ & 75 \\
\hline 4 & $*(.032)$ & .332 & $9^{*+*}(.024)$ & 347 & $3^{*+*}(.030)$ & .388 & $8^{*+*}(.034)$ & .335 & $65^{5+*}(.037)$ & .319 & $37^{7+}(.019)$ & 345 \\
\hline 5 & $*(.035)$ & .310 & $4^{* *}(.027)$ & .482 & $6^{* *}(.034)$ & .379 & $97^{* *}(.036)$ & .356 & $29^{* *}(.038)$ & .395 & $.641^{* *}(.022)$ & .412 \\
\hline \multicolumn{13}{|l|}{ RLS } \\
\hline s & & .170 & & .327 & & .306 & & 238 & & 263 & 022) & .270 \\
\hline 7 & 55) & .305 & 22) & .445 & 0) & .379 & 1) & .408 & 38) & 347 & $29 *(.019)$ & 395 \\
\hline 8 & 34) & .287 & $7^{\text {t* }}(.024)$ & .445 & 5) & .375 & 30) & .354 & $04^{* *}(.037)$ & .364 & $21^{* *}(.020)$ & 386 \\
\hline 9 & $9^{* * *}(.032)$ & .323 & $8^{* *}(.023)$ & .395 & $608^{* *}(.029)$ & .369 & $99^{* *}(.034)$ & .347 & $20^{* *}(.034)$ & .384 & $02^{* *}(.019)$ & .363 \\
\hline 10 & $0^{+* *}(.032)$ & .349 & $3^{*+*}(.021)$ & \begin{tabular}{|l|}
.481 \\
\end{tabular} & $642^{*+1}(.028)$ & .412 & $17^{7 *}(.033)$ & .380 & $.671^{* *}(.031)$ & .450 & $.664^{*+}(.019)$ & .441 \\
\hline \multicolumn{13}{|l|}{ RDS } \\
\hline 11 & $35)$ & .318 & & \begin{tabular}{|l|}
.373 \\
\end{tabular} & & .405 & & .272 & & .341 & .020) & $33^{3}$ \\
\hline & & .300 & 21) & \begin{tabular}{|l|}
.451 \\
\end{tabular} & $646^{*+}$ & 417 & 543 & 295 & & .447 & & 37 \\
\hline 1 & $5^{\text {t* }}(.031)$ & .354 & $1^{* *}(.019)$ & .437 & $.675^{* *}(.024)$ & .455 & & .321 & $.603^{3 *}(.035)$ & 364 & $.626^{* *}(.017)$ & 392 \\
\hline 14 & $.606^{t+1}(.033)$ & .368 & $0^{t+1}(.024)$ & .360 & $633^{*+1}(.029)$ & .401 & 34) & .320 & $.515^{+*}(.042)$ & .266 & $.581^{*+*}(.020)$ & .337 \\
\hline 15 & $.488^{+*}(.040)$ & .238 & $.596^{\text {t+ }}(.023)$ & .356 & $617^{* *}(.030)$ & 381 & $.557^{\text {t* }}(.036)$ & .310 & $.494^{* *}(.043)$ & .244 & $.554^{* *}(.021)$ & 307 \\
\hline
\end{tabular}




\begin{tabular}{|c|c|c|c|c|c|c|c|c|c|c|c|c|}
\hline \multirow[b]{2}{*}{ Items } & \multicolumn{2}{|l|}{ Male } & \multicolumn{2}{|l|}{ Female } & \multicolumn{2}{|l|}{ Grade 7} & \multicolumn{2}{|l|}{ Grade 8} & \multicolumn{2}{|l|}{ Grade 9} & \multicolumn{2}{|l|}{ Total } \\
\hline & SE) & $2^{2}$ & & $\mathrm{R}^{2}$ & & $\mathrm{R}^{2}$ & & $R^{2}$ & & $R^{2}$ & & $\mathrm{R}^{2}$ \\
\hline \multicolumn{13}{|l|}{ RCDS } \\
\hline 16 & & & & & & & & & & & & \\
\hline 1 & |f & 74 & 2) & & t & 64 & 1 & 317 & 770 & & 5 & 63 \\
\hline 18 & ** $(.029)$ & .375 & 20) & .425 & 8 & 87 & 0) & .341 & 30) & $\angle 2$ & (n+t) & 92 \\
\hline 19 & Fen & .302 & $5^{\text {t* }}(.023)$ & .390 & $2^{* *}(.029)$ & 25 & & 87 & 36) & 21 & $4^{*+*}($ & 53 \\
\hline $20+2$ & ) & 71 & 1) & 35 & tit & .415 & & 257 & & 317 & $7=1$ & .344 34 \\
\hline \multicolumn{13}{|l|}{$\overline{\mathrm{ODS}}$} \\
\hline 21 & 5) & 32 & & 2 & 4) & 11 & 8) & .392 & 40) & 27 & 20 & 51 \\
\hline 22 & 3) & 18 & 22) & t & $3^{* *}(.024)$ & .480 & 32) & .360 & 36) & 02 & $40 "$ & 10 \\
\hline 23 & 38) & .262 & 2) & 94 & 7) & .430 & $35)$ & .305 & 37) & 306 & 20) & 5 \\
\hline 24 & $.494^{* *}(.036)$ & .244 & $9^{* * *}(.023)$ & .359 & $0^{*+1}(.025)$ & .476 & 34) & 251 & $40^{* *}(.039)$ & 292 & $32^{2 *}($ & 38 \\
\hline 25 & $4(038)$ & 2 & $3^{*+*}(.022)$ & 34 & $5^{* *}(.027)$ & .442 & $02+3$. & .252 & $.532^{\text {t* }}(.038)$ & 283 & $78^{* *}(.020)$ & .00 \\
\hline \multicolumn{13}{|l|}{ RCS } \\
\hline 26 & & & & & & & & .321 & & & & $3 \varepsilon$ \\
\hline 27 & 29) & .356 & 18) & \begin{tabular}{|l|}
.496 \\
\end{tabular} & $0^{+*+}(.023)$ & .462 & 32) & .438 & $33)$ & & 17) & 4 \\
\hline 28 & $.547^{\text {t* }}(.034)$ & .299 & $5^{* *}(.020)$ & .470 & $2^{* *+}(.029)$ & .400 & $18^{* *}(.035)$ & .382 & 30) & 39 & $33^{3 *+}(.018)$ & .400 \\
\hline $2 \varsigma$ & $.560^{* *}(.029)$ & .314 & $0^{*+*}(.019)$ & \begin{tabular}{|l|}
.476 \\
\end{tabular} & $3^{*+*}(.029)$ & .426 & $02^{2+*}(.034)$ & .362 & $38^{+*}(.030)$ & 408 & $88^{* *}(.017)$ & .432 \\
\hline 30 & $610^{*+*}$ (0201 & .382 & & .403 & $6^{* *}(.027)$ & .443 & 36) & .315 & $.580^{* *}(.030)$ & .337 & $.626^{* *}(.019)$ & o \\
\hline \multicolumn{13}{|l|}{ RPPS } \\
\hline 31 & & 5 & & & & .42 & & $42-2+2$ & & & & 17 \\
\hline 3 & a) & .389 & $1^{\text {th }}$ & 463 & $0^{* *}$ & .51 & 1) & .410 & & & & 380 \\
\hline 33 & $.593^{\text {t* }}(.029)$ & .351 & $8^{* *}(.020)$ & \begin{tabular}{|l|}
.487 \\
\end{tabular} & $672^{* *}(.028)$ & .452 & $.685^{* *}(.026)$ & .469 & $.640^{* *}(.031)$ & 10 & $45^{*+}(.018)$ & .41 \\
\hline 3 & $.656^{\text {t+ }}(.030)$ & .431 & $.696^{*+}(.020)$ & .485 & $.699^{\text {** }}(.026)$ & .489 & $.602^{\text {t* }}(.032)$ & .363 & $.679^{* *}(.031)$ & 61 & $.679^{* *}(.017)$ & .46 \\
\hline 35 & $.617^{\text {t** }}(.037)$ & .380 & $.643^{3+*}(.022)$ & .413 & $.629^{\text {**t }}(.015)$ & .341 & $.567^{7 *}(.036)$ & 321 & $.618^{*+*}(.037)$ & .381 & $.607^{7+*}(.020)$ & .00 \\
\hline
\end{tabular}

Table 4. Factor correlation for 7 factors CFA of RSI

\begin{tabular}{|c|c|c|c|c|c|c|c|c|c|c|c|c|c|c|c|c|c|c|c|c|c|}
\hline Model & \multicolumn{7}{|c|}{ Male } & \multicolumn{7}{|c|}{ Female } & \multicolumn{7}{|c|}{ Total } \\
\hline Factor & 1. & 2. & 3. & 4. & 5. & 6. & 7 & 1. & 2. & 3. & 4. & 5. & 6. & 7. & 1. & 2. & 3. & 4. & 5. & 6. & \\
\hline $1 . R Q S$ & 1 & & & & & & & 1 & & & & & & & 1 & & & & & & \\
\hline 2.RLS & $.805^{\text {t* }}$ & 1 & & & & & & $.712^{2 *}$ & 1 & & & & & & $.724^{4 *}$ & 1 & & & & & \\
\hline 3.RDS & $.713^{* *}$ & $.813^{\text {t* }}$ & 1 & & & & & $.735^{\text {t* }}$ & $.904^{* *}$ & 1 & & & & & $.739^{\text {t*t }}$ & $.880^{*+*}$ & 1 & & & & \\
\hline 4.RCDS & $.744^{* *}$ & $.836^{\text {t+ }}$ & $.833^{\text {t*x }}$ & 1 & & & & $.713^{3+*}$ & $.803^{*+x}$ & . $951^{*+}$ & 1 & & & & $.726^{\text {t* }}$ & $.807^{\text {th }}$ & $935^{* *}$ & 1 & & & \\
\hline 5. RODS & $.822^{* *}$ & $.829^{*+1}$ & $.812^{*+}$ & $.945^{*+*}$ & 1 & & & $.768^{2+1}$ & $.771^{* *}$ & \begin{tabular}{|l|}
$.929^{* *}$ \\
\end{tabular} & $.945^{5 *}$ & 1 & & & $.762^{2 *}$ & $.762^{* *}$ & $.893^{*+x}$ & $.926^{* t}$ & 1 & & \\
\hline 6.RCS & $.696^{* *}$ & $.766^{*+}$ & $.741^{* *}$ & $.787^{* *}$ & $.904^{4+*}$ & 1 & & $.681^{*+}$ & $.764^{*+}$ & $.868^{* *+}$ & $.885^{* *}$ & $.933^{+*}$ & 1 & & $.687^{\text {t* }}$ & $.754^{* *}$ & $.842^{+*}$ & $.853^{\text {t* }}$ & . $885^{* * 4}$ & 1 & \\
\hline RRPS & $.720^{* *}$ & $.725^{\text {t* }}$ & $.712^{* *}$ & $.751^{* *}$ & $.826^{*+1}$ & $.824^{*+}$ & & $.665^{*+}$ & $.696^{+*}$ & $\mid .798^{*+}$ & $.861^{*+}$ & \begin{tabular}{|l|}
$.917^{4 k}$ \\
\end{tabular} & $.910^{*+*}$ & & $.705^{*+}$ & $.719^{*+1}$ & $.817^{* * 4}$ & $.856^{t+4}$ & \begin{tabular}{|l|}
$.901^{* *}$ \\
\end{tabular} & $.898^{\text {t* }}$ & 1 \\
\hline Model & & & & ade 7 & & & & & & & ade 8 & & & & & & & ade 9 & & & \\
\hline Factor & 1. & 2. & 3. & 4. & 5. & 6. & 7. & 1. & 2. & 3. & 4. & 5. & 6. & 7. & 1. & 2. & 3. & 4. & 5. & & \\
\hline 1.RQS & 1 & & & & & & & 1 & & & & & & & 1 & & & & & & \\
\hline 2.RLS & $.795^{\text {t* }}$ & 1 & & & & & & $.756^{*+}$ & 1 & & & & & & $.730^{*+}$ & 1 & & & & & \\
\hline 3.RDS & $.770^{* *}$ & $.947^{*+}$ & 1 & & & & & $.783^{3+\infty}$ & $891^{\text {** }}$ & 1 & & & & & $.744^{*+}$ & $.840^{*+4}$ & 1 & & & & \\
\hline 4.RCDS & $.714^{* *}$ & $.866^{*+}$ & $.934^{* *}$ & 1 & & & & $.758^{*+1}$ & $763^{+*}$ & $.969^{*+4}$ & 1 & & & & $.727^{7 *}$ & $.761^{*+}$ & $.878^{*+1}$ & 1 & & & \\
\hline 5.RODS & $.810^{\text {t* }}$ & $.904^{*+}$ & $.915^{* *}$ & $.930^{*+}$ & 1 & & & $.782^{2+}$ & $.694^{+*}$ & $.878^{*+}$ & $.924^{*+}$ & 1 & & & $.719^{\text {t* }}$ & $.673^{\text {t* }}$ & $.791^{* *}$ & $.858^{*+4}$ & 1 & & \\
\hline 6.RCS & $.750^{* *}$ & $.904^{* *}$ & $.923^{*+4}$ & $.871^{*+*}$ & $.928^{*+*}$ & 1 & & $.715^{\text {t* }}$ & $.751^{*+}$ & \begin{tabular}{|l|}
$.858^{* *}$ \\
\end{tabular} & $.934^{*+}$ & $\mid 946^{* *+}$ & 1 & & $.695^{*+}$ & $.654^{* *}$ & $.770^{*+}$ & $.776^{+4}$ & $.799^{* *}$ & 1 & \\
\hline 7.RRPS & $.722^{* *}$ & $.811^{*}$ & $.850^{*+}$ & $.863^{\text {tw }}$ & $.875^{5 *}$ & $.921^{t *}$ & & $.710^{* *}$ & $.618^{+*+}$ & $.740^{* *}$ & $.758^{* *}$ & $.898^{* *}$ & $.875^{+*}$ & & $.698^{* *}$ & $.673^{*+}$ & $.752^{* *}$ & $.844^{*+x}$ & $.863^{* *}$ & $.856^{*+}$ & \\
\hline
\end{tabular}


Table 5. Measurement invariance summary fit statistics across gender

\begin{tabular}{|c|c|c|c|c|c|c|c|c|c|c|c|c|c|}
\hline & Model & Comparison & $x^{2}$ & $\begin{array}{l}x^{2} \text { degree of } \\
\text { freedom }\end{array}$ & CFI & TLI & SRMR & RMSEA & $\begin{array}{l}\text { RMSEA } \\
90 \% \text { C.I. }\end{array}$ & $\Delta x^{2}$ & $\Delta d f$ & $\Delta \mathrm{CFI}$ & $\begin{array}{c}x^{2} \text { difference test } \\
\text { (significance value) }\end{array}$ \\
\hline & $\begin{array}{l}\text { Configural } \\
\text { invariance }\end{array}$ & & & & & & & & & & & & \\
\hline & $\begin{array}{l}\text { Male baseline } \\
\text { model }\left(\mathrm{M}_{1}\right)\end{array}$ & - & 1058.210 & 535 & 0.916 & 0.906 & 0.038 & 0.039 & {$[0.036,0.043]$} & - & - & - & - \\
\hline & $\begin{array}{l}\text { Female baseline } \\
\text { model }\left(\mathrm{M}_{2}\right)\end{array}$ & - & 1413.328 & 536 & 0.942 & 0.935 & 0.030 & 0.039 & {$[0.037,0.042]$} & - & - & - & - \\
\hline & $\begin{array}{l}\text { Testing configural } \\
\text { invariance }\left(\mathrm{M}_{3}\right)\end{array}$ & - & 2471.538 & 1071 & 0.934 & 0.927 & 0.033 & 0.039 & {$[0.037,0.041]$} & - & - & - & - \\
\hline & $\begin{array}{l}\text { Weak measurement } \\
\text { invariance }\left(\mathrm{M}_{4}\right)\end{array}$ & & 2498.046 & 1099 & 0.934 & 0.929 & 0.035 & 0.039 & {$[0.037,0.041]$} & & & & \\
\hline 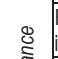 & $\begin{array}{l}\begin{array}{l}\text { Factor loading } \\
\text { invariance }\end{array} \\
\end{array}$ & $\mathrm{M}_{4}-\mathrm{M}_{3}$ & & & & & & & & 26.538 & 28 & - & 0.543 \\
\hline 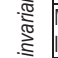 & $\begin{array}{l}\text { Maker items' factor } \\
\text { loading invariance }\end{array}$ & $M_{5}-M_{6}$ & - & - & - & - & - & - & - & 6.128 & 7 & & 0.523 \\
\hline 竎 & $\begin{array}{l}\text {-without equality } \\
\text { restriction on factor } \\
\text { loading }\left(M_{5}\right)\end{array}$ & - & 2471.538 & 1071 & 0.934 & 0.927 & 0.033 & 0.039 & $\mid[0.037,0.041]$ & - & - & - & - \\
\hline है & \begin{tabular}{|l|} 
- with equality \\
restriction on factor \\
loading $\left(M_{6}\right)$
\end{tabular} & - & 2477.666 & 1078 & 0.934 & 0.927 & 0.033 & 0.039 & {$[0.037,0.041]$} & - & - & - & - \\
\hline$\stackrel{\infty}{\infty}$ & Strong & & 2586.109 & 1134 & 0.932 & 0.928 & 0.036 & \begin{tabular}{|l|}
0.039 \\
\end{tabular} & {$[0.037,0.041]$} & & & & \\
\hline & $\begin{array}{l}\text { measurement } \\
\text { invariance } \\
\text { (invariance of factor } \\
\text { loadings and item } \\
\text { intercepts })\left(\mathrm{M}_{7}\right) \\
\end{array}$ & $\mathrm{M}_{7}-\mathrm{M}_{3}$ & & & & & & & & 114.571 & 63 & 0.002 & 0.000 \\
\hline & $\begin{array}{l}\text { Strict measurement } \\
\text { invariance } \\
\text { (error variance } \\
\text { invariance) }\left(\mathrm{M}_{8}\right)\end{array}$ & & 2866.978 & 1169 & 0.920 & 0.919 & 0.050 & 0.041 & {$[0.039,0.043]$} & & & & \\
\hline & \begin{tabular}{|l} 
- no equality \\
restriction on item \\
invariance
\end{tabular} & $\mathrm{M}_{8}-\mathrm{M}_{3}$ & & & & & & & & 395.44 & 98 & - & 0.000 \\
\hline & \begin{tabular}{|l|} 
- equality restriction \\
on item invariance
\end{tabular} & $\mathrm{M}_{8}-\mathrm{M}_{7}$ & - & - & - & - & - & - & - & 280.869 & 35 & - & 0.000 \\
\hline & \begin{tabular}{|l|} 
Factor variance \\
invariance $\left(\mathrm{M}_{\mathrm{g}}\right)$
\end{tabular} & & 2607.808 & 1141 & 0.931 & 0.928 & 0.046 & 0.039 & {$[0.037,0.041]$} & & & & \\
\hline ్ㅠㄴ & & $\mathrm{M}_{9}-\mathrm{M}_{3}$ & & & & & & & & 136.270 & 70 & .003 & 3.653 \\
\hline 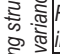 & $\begin{array}{l}\text { Factor covariance } \\
\text { invariance }\left(\mathrm{M}_{10}\right)\end{array}$ & & 2634.134 & 1155 & 0.931 & 0.928 & 0.047 & 0.039 & {$[0.037,0.041]$} & & & & \\
\hline ही & & $\mathrm{M}_{10}-\mathrm{M}_{3}$ & & & & & & & & 162.596 & 84 & .003 & 5.976 \\
\hline & \begin{tabular}{|l|} 
Factor mean \\
invariance
\end{tabular} & $\begin{array}{l}\text { QS(-0.034 } \\
061, \mathrm{P}=0.3\end{array}$ & $\begin{array}{l}\text { 5), RLS } \\
\text { RPS(-0 }\end{array}$ & $\begin{array}{l}\mathrm{P}=0.997 \\
=0.003)\end{array}$ & & $\overline{0, P}$ & 878 & & $40, P=0$. & $\mathrm{ROL}$ & $(-0$. & $P=$ & (- \\
\hline
\end{tabular}

Results of structural invariance show that $\Delta x^{2}(\mathrm{df}=70)=136.270, p=3.653$ which is not statistically significant, $\Delta \mathrm{CFI}=0.003<0.01$. Therefore factor variance invariance across gender. Finding covariance shows that $\Delta x^{2}(\mathrm{df}=84)=$ 162.596, $p=5.976$ which is not statistically significant, $\Delta \mathrm{CFI}=0.003<0.01$. Therefore factor covariance invariance across gender. Finally, findings showed that RQS $(-0.034, p=0.557), \operatorname{RLS}(0.000, p=0.997), \operatorname{RDS}(-0.010, p=0.878), \operatorname{RCDS}(-$ $0.040, p=0.504)$, RCS $(-0.061, p=0.314)$ are significantly lower in females than in males, while the factor mean of RODS $(-0.150, p=0.015)$ and RRPS $(-0.178, p=0.003)$ are not significantly different between the gender.

\subsubsection{Measurement Invariance across Grade 7 and Grade 8}

The model fit similarity for Grade 7 and Grade 8 as such $\chi^{2}(\mathrm{df}=535)=1022.911, p<0.001 ; \mathrm{CFI}=.948$; TLI= .942; SRMR= .031 , RMSEA $=.038$; RMSEA 90\% C.I. $=[.034, .041]$ for Grade 7; and $X^{2}(\mathrm{df}=533)=990.289, p<0.001 ; \mathrm{CFI}=.926$; TLI= .917 ; SRMR $=.038$, RMSEA $=.039$; RMSEA 90\% C.I. $=[.035, .042]$ for Grade 8 . The Grade 7 and Grade 8 baseline models for analyzing configural model fit the data well: $X^{2}(\mathrm{df}=1071)=2013.201, p<0.001 ; \mathrm{CFI}=.939 ; \mathrm{TLI}=.932$; SRMR= .034 , RMSEA= .038; RMSEA 90\% C.I. = [.035,.041] (Table 6).

Weak measurement indicates of testing metric invariance is $\Delta x^{2}(\mathrm{df}=28)=53.487, p=0.003$ which is statistically 
significant. Since one of the factor loading of each factor or 'marker items', including item 1, item 6, item 11, item 16, item 21, item 26, and item 31, are not tested in the LR test so they must be tested invariance of the marker items factor loading. The difference in model $x^{2}$ statistics between model with and without equality restriction; $\Delta x^{2}(\mathrm{df}=7)=18.942$, $p=0.008$ which is statistically significant, indicating that the factor loading of maker items are non-invariance across Grade 7 and Grade 8.

Strong measurement with LR test of scalar invariance shows as such $\Delta x^{2}(\mathrm{df}=63)=178.300, p=5.915$ which is not statistically significant but $\Delta \mathrm{CFI}=0.007$ is smaller than 0.01 . Thus, both factor loading and item intercept of the RSI are variance across Grade 7 and Grade 8 . Strict measurement with LR test of two nested models of error invariance are $\Delta x^{2}$ $(\mathrm{df}=98)=382.073, p=0.000$ of no equality restriction on item invariance which are statistically significant. The corresponding $\Delta x^{2}(\mathrm{df}=35)=203.773, p=0.000$ of equality restriction on item invariance which are statistically significant. Thus, results indicate that item invariances are non-invariant across Grade 7 and Grade 8.

Results of structural invariance show that $\Delta x^{2}(\mathrm{df}=70)=188.068, p=9.420$ which is not statistically significant, $\Delta \mathrm{CFI}=0.007<0.01$. Therefore factor variance invariance across Grade 7 and Grade 8. Finding covariance shows that $\Delta x^{2}(\mathrm{df}=84)=219.939, p=4.052$ which is not statistically significant, $\Delta \mathrm{CFI}=0.009<0.01$. Therefore factor covariance invariance across Grade 7 and Grade 8 . Finally, findings showed that $\operatorname{RQS}(0.210, p=0.000), \operatorname{RLS}(0.075, p=0.011), \operatorname{RDS}$ $(0.074, p=0.022), \operatorname{RCDS}(0.128, p=0.000), \operatorname{RODS}(0.143, p=0.000), \operatorname{RCS}(0.160, p=0.000)$ are significantly higher in Grade 8 than in Grade 7.

\subsubsection{Measurement Invariance across Grade 8 and Grade 9}

The model fit similarity for Grade 8 and Grade 9 as such $X^{2}(d f=533)=990.289, p<0.001 ; C F I=.926 ;$ TLI= .917; SRMR= .038 , RMSEA = .039; RMSEA 90\% C.I. $=[.035, .042]$ for Grade 8; and $X^{2}(\mathrm{df}=531)=880.814, p<0.001 ; \mathrm{CFI}=.936$; TLI= .929 ; SRMR $=.038$, RMSEA $=.037$; RMSEA 90\% C.I. $=[.033, .041]$ for Grade 9. The Grade 8 and Grade 9 baseline models for analyzing configural model fit the data well: $X^{2}(\mathrm{df}=1064)=1878.104, p<0.001 ; \mathrm{CFI}=.931 ; \mathrm{TLI}=.923$; SRMR= .038, RMSEA $=.038 ;$ RMSEA 90\% C.I. $=[.035, .041]$ (Table 7).

Weak measurement indicates of testing metric invariance is $\Delta x^{2}(\mathrm{df}=28)=21.196, p=0.817$ which is not statistically significant. Since one of the factor loading of each factor or 'marker items', including item 1, item 6 , item 11 , item 16, item 21, item 26, and item 31, are not tested in the LR test so they must be tested invariance of the marker items factor loading. The difference in model $x^{2}$ statistics between model with and without equality restriction; $\Delta x^{2}(\mathrm{df}=7)=$ 3.154, $p=0.870$ which is not statistically significant, indicating that the factor loading of maker items are invariance across Grade 8 and Grade 9.

Strong measurement with LR test of scalar invariance shows as such $\Delta x^{2}(\mathrm{df}=63)=94.771, p=0.006$ which is statistically significant but $\Delta \mathrm{CFI}=0.003$ is smaller than 0.01 . Thus, both factor loading and item intercept of the RSI are non-variance across Grade 8 and Grade 9. Strict measurement with LR test of two nested models of error invariance are $\Delta x^{2}(\mathrm{df}=98)=139.157, p=0.004$ of no equality restriction on item invariance which are statistically significant. The corresponding $\Delta x^{2}(\mathrm{df}=35)=44.386, p=0.134$ of equality restriction on item invariance which are not statistically significant. Thus, results indicate that item variances are non-invariant across groups.

Results of structural invariance show that $\Delta x^{2}(\mathrm{df}=70)=97.911, p=0.015$ which is statistically significant, $\Delta \mathrm{CFI}=0.002<0.01$. Therefore factor variance non-invariance across Grade 8 and Grade 9 . Finding covariance shows that $\Delta x^{2}(\mathrm{df}=84)=119.140, p=0.007$ which is statistically significant, $\Delta \mathrm{CFI}=0.003<0.01$. Therefore factor covariance noninvariance across Grade 8 and Grade 9. Finally, findings showed that RLS $(-0.080, p=0.014), \operatorname{RDS}(-0.093, p=0.009)$, $\operatorname{RCDS}(-0.095, p=0.017), \operatorname{RODS}(-0.090, p=0.029), \operatorname{RCS}(-0.145, p=0.000)$, and RRPS $(-0.192, p=0.000)$, are significantly lower in Grade 8 than in Grade 9 while the factor mean of RQS $(-0.057, p=0.064)$ are not significantly different between the two samples.

\subsubsection{Measurement Invariance across Grade 7 and Grade 9}

The ML was used for testing measurement invariance. The model fit similarity for Grade 7 and Grade 9 as such $X^{2}$ $(\mathrm{df}=535)=1022.911, p<0.001 ; \mathrm{CFI}=.948 ; \mathrm{TLI}=.942$; SRMR $=.031$, RMSEA $=.038$; RMSEA $90 \%$ C.I. $=[.034, .041]$ for Grade 7; and $X^{2}(\mathrm{df}=531)=887.814, p<0.001 ; \mathrm{CFI}=.936$; TLI= .929; SRMR= .038, RMSEA $=.037$; RMSEA 90\% C.I. = $[.033, .041]$ for Grade 9 . The Grade 7 and Grade 9 baseline models for analyzing configural model fit the data well: $X^{2}$ $(\mathrm{df}=1066)=1910.726, p<0.001 ; \mathrm{CFI}=.944 ; \mathrm{TLI}=.937$; SRMR $=.035$, RMSEA $=.037$; RMSEA 90\% C.I.= [.035,.040] (Table 8). 
Weak measurement indicates of testing metric invariance is $\Delta x^{2}(\mathrm{df}=28)=28.631, p=0.431$ which is not statistically significant. Since one of the factor loading of each factor or 'marker items', including item 1, item 6 , item 11 , item 16, item 21, item 26, and item 31, are not tested in the LR test so they must be tested invariance of the marker items factor loading. The difference in model $x^{2}$ statistics between model with and without equality restriction; $\Delta x^{2}(\mathrm{df}=7)=$ $6.7, p=0.461$ which is not statistically significant, indicating that the factor loading of maker items are invariance across Grade 7 and Grade 9.

Table 6. Measurement invariance summary fit statistics across grade 7 and grade 8

\begin{tabular}{|c|c|c|c|c|c|c|c|c|c|c|c|c|c|}
\hline \multicolumn{2}{|r|}{ Model } & Comparison & $x^{2}$ & $\begin{array}{c}x^{2} \text { degree } \\
\text { of } \\
\text { freedom }\end{array}$ & $\mathrm{CFI}$ & TLI & SRMR & RMSEA & $\begin{array}{l}\text { RMSEA } \\
90 \% \text { C.I. }\end{array}$ & $\Delta x^{2}$ & $\Delta d f$ & $\Delta \mathrm{CFI}$ & $\begin{array}{c}x^{2} \text { difference test } \\
\text { (significance } \\
\text { value) }\end{array}$ \\
\hline \multirow{14}{*}{ 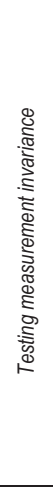 } & Configural invariance & & & & & & & & & & & & \\
\hline & Grade 7 baseline model $\left(M_{1}\right)$ & - & 1022.911 & 535 & 0.948 & 0.942 & 0.031 & 0.038 & {$[0.034,0.041]$} & - & - & - & - \\
\hline & Grade 8 baseline model $\left(\mathrm{M}_{2}\right)$ & - & 990.289 & 533 & 0.926 & 0.917 & 0.038 & 0.039 & {$[0.035,0.042]$} & - & - & - & - \\
\hline & Testing configural invariance $\left(\mathrm{M}_{3}\right)$ & - & 2013.201 & 1068 & 0.939 & 0.932 & 0.034 & 0.038 & {$[0.035,0.041]$} & - & - & - & - \\
\hline & Weak measurement invariance $\left(M_{4}\right)$ & & 2066.688 & 1096 & 0.938 & 0.932 & 0.039 & 0.038 & {$[0.036,0.041]$} & & & & \\
\hline & Factor loading invariance & $\mathrm{M}_{4}-\mathrm{M}_{3}$ & - & - & - & - & - & - & - & 53.487 & 28 & - & .003 \\
\hline & Maker items' factor loading invariance & $M_{5}-M_{6}$ & - & - & - & - & - & - & - & 18.942 & 7 & - & .008 \\
\hline & \begin{tabular}{|l}
- without equality restriction on factor loading \\
$\left(M_{5}\right)$
\end{tabular} & - & 2013.201 & 1068 & 0.939 & $0.932 \mid$ & 0.034 & 0.038 & {$[0.035,0.041]$} & - & - & - & - \\
\hline & $\begin{array}{l}\text { with equality restriction on factor loading } \\
\left(M_{6}\right)\end{array}$ & - & 2032.143 & 1075 & 0.938 & $0.932 \mid$ & 0.036 & 0.038 & $[0.036,0.041]]$ & - & - & - & - \\
\hline & Strong measurement invariance & - & 2191.501 & 1131 & 0.932 & 0.928 & 0.046 & 0.039 & {$[0.037,0.042]$} & & & & \\
\hline & $\begin{array}{l}(\text { Invarlance of factor loadings and Item } \\
\text { intercepts ) }\left(\mathrm{M}_{7}\right)\end{array}$ & $\mathrm{M}_{7}-\mathrm{M}_{3}$ & & - & - & - & & & & 178.3 & 63 & .007 & 5.915 \\
\hline & $\begin{array}{l}\text { Strict measurement invariance } \\
\text { (error variance invariance) }\left(M_{8}\right)\end{array}$ & - & 2395.274 & 1166 & 0.921 & 0.919 & 0.049 & 0.042 & $[0.039,0.044]]$ & - & - & - & - \\
\hline & - no equality restriction on item invariance & $M_{8}-M_{3}$ & - & - & - & - & - & - & - & 382.073 & 98 & - & .000 \\
\hline & - equality restriction on item invariance & $\mathrm{M}_{8}-\mathrm{M}_{7}$ & - & - & - & - & - & - & - & 203.773 & 35 & - & .000 \\
\hline \multirow{5}{*}{ 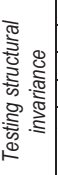 } & Factor variance invariance $\left(\mathrm{M}_{9}\right)$ & - & 2201.269 & 1138 & 0.932 & 0.928 & 0.053 & 0.039 & {$[0.037,0.042]$} & - & 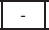 & - & - \\
\hline & & $\mathrm{Mg}_{9}-\mathrm{M}_{3}$ & - & - & - & - & - & - & - & 188.068 & 70 & 007 & 9.420 \\
\hline & Factor covariance invariance $\left(\mathrm{M}_{10}\right)$ & - & 2233.140 & 1152 & 0.930 & 0.928 & 0.058 & 0.039 & {$[0.037,0.042]$} & - & - & - & - \\
\hline & & $\mathrm{M}_{10}-\mathrm{M}_{3}$ & - & - & - & - & & - & - & 219.939 & 84 & .009 & 4.052 \\
\hline & Factor mean invariance & \multicolumn{12}{|c|}{$\begin{array}{l}\operatorname{RQS}(0.210, P=0.000), \operatorname{RLS}(0.075, P=0.011), \operatorname{RDS}(0.074, P=0.022), \operatorname{RCDS}(0.128, P=0.000) \\
\operatorname{RODS}(0.143, P=0.000), \operatorname{RCS}(0.160, P=0.000), \operatorname{RRPS}(0.168, P=0.000)\end{array}$} \\
\hline
\end{tabular}

Table 7. Measurement invariance summary fit statistics across grade 8 and grade 9

\begin{tabular}{|c|c|c|c|c|c|c|c|c|c|c|c|c|c|}
\hline & Model & Comparison & $x^{2}$ & $\begin{array}{l}x^{2} \text { degree of } \\
\text { freedom }\end{array}$ & $\mathrm{CFI}$ & TLI & SRMR & RMSEA & $\begin{array}{l}\text { RMSEA } \\
90 \% \text { C.I. }\end{array}$ & $\Delta x^{2}$ & $\Delta d f$ & $\Delta \mathrm{CFI}$ & $\begin{array}{l}x^{2} \text { difference test } \\
\text { (significance } \\
\text { value) }\end{array}$ \\
\hline \multirow{13}{*}{ 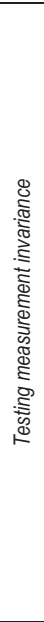 } & Configural invariance & & & & & & & & & & & & \\
\hline & Grade 8 baseline model $\left(M_{1}\right)$ & - & 990.289 & 533 & 0.926 & 0.917 & 0.038 & 0.039 & {$[0.035,0.042]$} & - & - & - & - \\
\hline & Grade 9 baseline model $\left(\mathrm{M}_{2}\right)$ & - & 887.814 & 531 & 0.936 & 0.929 & 0.038 & 0.037 & {$[0.033,0.041]$} & - & - & - & - \\
\hline & $\begin{array}{l}\text { Testing configural invariance } \\
\left(M_{3}\right)\end{array}$ & - & 1878.104 & 1064 & 0.931 & 0.923 & 0.038 & 0.038 & {$[0.035,0.041]$} & - & - & - & - \\
\hline & $\begin{array}{l}\text { Weak measurement } \\
\text { invariance }\left(\mathrm{M}_{4}\right)\end{array}$ & - & 1899.300 & 1092 & 0.931 & 0.925 & 0.040 & 0.037 & {$[0.034,0.040]$} & & & & \\
\hline & Factor loading invariance & $M_{4}-M_{3}$ & - & - & - & - & - & - & - & 21.196 & 28 & - & .817 \\
\hline & $\begin{array}{l}\text { Maker items' factor loading } \\
\text { invariance }\end{array}$ & $M_{5}-M_{6}$ & - & - & - & - & - & - & - & 3.154 & 7 & - & .870 \\
\hline & $\begin{array}{l}\text {-without equality restriction on } \\
\text { factor loading }\left(\mathrm{M}_{5}\right)\end{array}$ & - & 1878.104 & 1064 & 0.931 & 0.923 & 0.038 & 0.038 & {$[0.035,0.041]$} & - & - & - & - \\
\hline & $\begin{array}{l}\text { - with equality restriction on } \\
\text { factor loading }\left(\mathrm{M}_{6}\right)\end{array}$ & - & 1881.253 & 1071 & 0.931 & 0.924 & 0.038 & 0.038 & {$[0.035,0.040]$} & - & - & - & - \\
\hline & $\begin{array}{l}\text { Strong measurement invariance } \\
\text { (invariance of factor loadings } \\
\text { and item intercepts ) }\left(\mathrm{M}_{7}\right)\end{array}$ & $\mathrm{M}_{7}-\mathrm{M}_{3}$ & 1972.875 & 1127 & 0.928 & 0.924 & 0.044 & 0.038 & {$[0.035,0.040]$} & 94.771 & 63 & .003 & .006 \\
\hline & \begin{tabular}{|l} 
Strict measurement invariance \\
(error variance invariance) $\left(M_{8}\right)$
\end{tabular} & - & 2017.261 & 1162 & 0.927 & 0.926 & 0.047 & 0.037 & {$[0.034,0.040]$} & - & - & - & - \\
\hline & $\begin{array}{l}\text { - no equality restriction on item } \\
\text { invariance }\end{array}$ & $M_{8}-M_{3}$ & - & - & - & - & - & - & - & 139.157 & 98 & - & .004 \\
\hline & $\begin{array}{l}\text { - equality restriction on item } \\
\text { invariance }\end{array}$ & $M_{8}-M_{7}$ & - & - & - & - & - & - & - & 44.386 & 35 & - & .134 \\
\hline
\end{tabular}




\begin{tabular}{|c|c|c|c|c|c|c|c|c|c|c|c|c|c|}
\hline & Model & Comparison & $x^{2}$ & $\begin{array}{c}x^{2} \text { degree of } \\
\text { freedom }\end{array}$ & $\mathrm{CFI}$ & TLI & SRMR & RMSEA & $\begin{array}{l}\text { RMSEA } \\
90 \% \text { C.I. }\end{array}$ & $\Delta x^{2}$ & $\Delta d f$ & $\Delta \mathrm{CFI}$ & $\begin{array}{l}x^{2} \text { difference test } \\
\text { (significance } \\
\text { value) }\end{array}$ \\
\hline & Factor variance invariance $\left(\mathrm{M}_{9}\right)$ & - & 1976.015 & 1134 & 0.929 & 0.925 & 0.046 & 0.037 & $|[0.035,0.040]|$ & - & - & - & 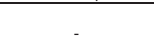 \\
\hline 竎 \& & & $M_{9}-M_{3}$ & - & - & - & - & - & - & - & 97.911 & 70 & .002 & .015 \\
\hline 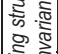 & $\begin{array}{l}\text { Factor covariance invariance } \\
\left(\mathrm{M}_{10}\right)\end{array}$ & - & 1997.244 & 1148 & 0.928 & 0.925 & 0.046 & 0.037 & $\mid[0.035,0.040]$ & - & - & - & - \\
\hline 氙. & & $\mathrm{M}_{10}-\mathrm{M}_{3}$ & - & 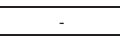 & -5 & - & - & - & - & 119.14 & 84 & .003 & .007 \\
\hline & Factor mean invariance & $\begin{array}{l}\mathrm{RQS}(-.057 \\
.145, \mathrm{P}=.00\end{array}$ & $\begin{array}{l}5=.064), \\
), \text { RRPS }\end{array}$ & $\begin{array}{l}80, P=.01 \\
D=.000)\end{array}$ & & & $.009)$, & $\mathrm{SS}(-$ & $P=.01$ & $\mathrm{ODS}(-. \mathrm{C}$ & $\overline{D P}$ & $.029)$ & CSS(- \\
\hline
\end{tabular}

Table 8. Measurement invariance summary fit statistics across grade 7 and grade 9

\begin{tabular}{|c|c|c|c|c|c|c|c|c|c|c|c|c|c|}
\hline & Model & Comparison & $x^{2}$ & $\begin{array}{l}x^{2} \text { degree of } \\
\text { freedom }\end{array}$ & $\mathrm{CFI}$ & TLI & SRMR & RMSEA & $\begin{array}{l}\text { RMSEA } \\
90 \% \text { C.I. }\end{array}$ & $\Delta x^{2}$ & $\Delta d f$ & $\Delta \mathrm{CFI}$ & $\begin{array}{c}x^{2} \text { difference test } \\
\text { (significance } \\
\text { value) }\end{array}$ \\
\hline \multirow{14}{*}{ 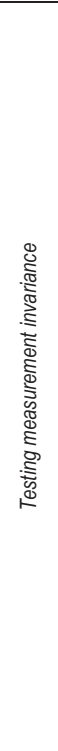 } & Configural invariance & & & & & & & & & & & & \\
\hline & Grade 7 baseline model $\left(M_{1}\right)$ & - & 1022.911 & 535 & 0.948 & 0.942 & 0.031 & 0.038 & {$[0.034,0.041]$} & - & - & - & - \\
\hline & Grade 9 baseline model $\left(\mathrm{M}_{2}\right)$ & - & 887.814 & 531 & 0.936 & 0.929 & 0.038 & 0.037 & {$[0.033,0.041]$} & - & - & - & - \\
\hline & $\begin{array}{l}\text { Testing configural invariance } \\
\left(M_{3}\right)\end{array}$ & - & $\mid 1910.726$ & 1066 & 0.944 & 0.937 & 0.035 & 0.037 & {$[0.035,0.040]$} & - & - & - & - \\
\hline & $\begin{array}{l}\text { Weak measurement } \\
\text { invariance }\end{array}$ & & $1939.357 \mid$ & 1094 & 0.943 & 0.939 & 0.037 & 0.037 & {$[0.034,0.040]$} & & & & \\
\hline & $\begin{array}{l}\text { Factor loading invariance } \\
\left(\mathrm{M}_{4}\right)\end{array}$ & $\mathrm{M}_{4}-\mathrm{M}_{3}$ & - & - & - & - & - & - & - & 28.631 & 28 & - & 431 \\
\hline & $\begin{array}{l}\text { Maker items' factor loading } \\
\text { invariance }\end{array}$ & $M_{5}-M_{6}$ & - & - & - & - & - & - & - & 6.7 & 7 & - & .461 \\
\hline & $\begin{array}{l}\text {-without equality restriction on } \\
\text { factor loading }\left(\mathrm{M}_{5}\right)\end{array}$ & - & $|1910.726|$ & 1066 & 0.944 & $0.937 \mid$ & 0.035 & 0.037 & {$[0.035,0.040]$} & - & - & - & - \\
\hline & $\begin{array}{l}\text { - with equality restriction on } \\
\text { factor loading }\left(\mathrm{M}_{6}\right)\end{array}$ & - & $|1917.426|$ & 1073 & 0.944 & $0.937 \mid$ & 0.035 & 0.037 & {$[0.035,0.040]$} & - & - & - & - \\
\hline & $\begin{array}{l}\text { Strong measurement } \\
\text { invariance } \\
\text { (invariance of factor loadings } \\
\text { and item intercepts })\left(M_{7}\right)\end{array}$ & - & $2068.080 \mid$ & 1129 & 0.937 & 0.934 & 0.040 & 0.038 & $|[0.036,0.041]|$ & 157.354 & 63 & .007 & 4.948 \\
\hline & & $\mathrm{M}_{7}-\mathrm{M}_{3}$ & - & - & - & - & - & - & - & 157.354 & 63 & .007 & 4.948 \\
\hline & \begin{tabular}{|l|} 
Strict measurement \\
invariance \\
$($ (error variance invariance) \\
$\left(M_{8}\right)$ \\
\end{tabular} & - & 2223.595 & 1164 & 0.929 & $0.928 \mid-2$ & 0.048 & 0.040 & {$[0.038,0.043]$} & - & - & - & - \\
\hline & $\begin{array}{l}- \text { no equality restriction on } \\
\text { item invariance }\end{array}$ & $\mathrm{M}_{8}-\mathrm{M}_{3}$ & & & & & & & & 312.869 & 98 & - & .000 \\
\hline & $\begin{array}{l}\text { - equality restriction on item } \\
\text { invariance }\end{array}$ & $\mathrm{M}_{8}-\mathrm{M}_{7}$ & - & - & - & - & - & - & - & 155.515 & 35 & - & .000 \\
\hline \multirow{3}{*}{ 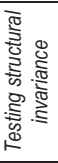 } & $\begin{array}{l}\text { Factor variance invariance } \\
\left(\mathrm{M}_{9}\right)\end{array}$ & $\mathrm{M}_{9}-\mathrm{M}_{3}$ & 2072.211 & 1136 & 0.937 & $0.934 \mid$ & 0.042 & 0.038 & {$[0.036,0.041]$} & 161.485 & 70 & .007 & 3.439 \\
\hline & $\begin{array}{l}\text { Factor covariance invariance } \\
\left(\mathrm{M}_{10}\right)\end{array}$ & $\mathrm{M}_{10}-\mathrm{M}_{3}$ & 2093.762 & 1150 & 0.937 & 0.935 & 0.047 & 0.038 & {$[0.035,0.041]$} & 183.036 & 84 & .004 & 2.530 \\
\hline & Factor mean invariance & \multicolumn{12}{|c|}{$\begin{array}{l}\text { RQS(.140,P=.000), RLS(-.007,P=.828), RDS(-.009,P=.795),RCDS(.034,P=.378), RODS(.055,P=.113), RCS(- } \\
.002, P=.950), \operatorname{RRPS}(-.012, P=.755)\end{array}$} \\
\hline
\end{tabular}

Strong measurement with LR test of scalar invariance shows as such $\Delta x^{2}(\mathrm{df}=63)=157.354, p=4.948$ which is not statistically significant but $\Delta \mathrm{CFI}=0.007$ is much smaller than 0.01 . Thus, both factor loading and item intercept of the RSI are variance across Grade 7 and Grade 9. Strict measurement with LR test of two nested models of error invariance are $\Delta x^{2}(\mathrm{df}=98)=312.869, p=0.000$ of no equality restriction on item invariance which are statistically significant. The corresponding $\Delta x^{2}(\mathrm{df}=35)=155.515, p=0.000$ of equality restriction on item invariance which are not statistically significant. Thus, results indicate that item variances are non-invariant across Grade 7 and Grade 9.

Results of structural invariance show that $\Delta x^{2}(\mathrm{df}=70)=161.485, p=3.439$ which is not statistically significant, $\Delta \mathrm{CFI}=0.007<0.01$. Therefore factor variance invariance across Grade 7 and Grade 9 . Finding covariance shows that $\Delta x^{2}(\mathrm{df}=84)=183.036, p=2.530$ which is not statistically significant, $\Delta \mathrm{CFI}=0.004<0.01$. Therefore factor covariance invariance across Grade 7 and Grade 9. Finally, findings showed that $\mathrm{RQS}(0.140, p=0.000)$ are significantly higher in Grade 9 than Grade 7. Meanwhile RLS, RDS, RCDS, RODS, RCS, and RRPS are not statistically significant different between the two groups of samples. 


\section{Discussion}

On this line of reasoning, RSs is considered as the most important because students who possessed high RSs are more likely to engage in learning outcomes and achievement at higher level (Zehra, Hassaan, \& Mushtaq, 2015). In addition, investigating CV and measurement invariance of RSs across gender and grade provide a further understanding of how RSs can be measured and evaluated.

This study was conducted purposively to examine the validity of the RSI with samples of Grade 7 to Grade 9 . The validity of research skills consist of male, female, Grade 7, Grade 8, Grade 9, and total CFA model which are the most well fit for all models. Results from this study provide evidence about the validity of the confirming RSI. Moreover, researchers investigate the measurement invariance across gender and grade. Conclusion is shown in Table 9.

Testing measurement invariance can show metric invariance is mostly invariance except metric invariance across Grade 7 and Grade 8 is non-invariance. In other word, factor loading is generally having same pattern except observed indicator variables measure is different factors across Grade 7 and Grade 8. Secondly, test scalar invariance is mostly invariance except scalar invariance across gender.

Table 9. Conclusion of measurement invariance testing

\begin{tabular}{|c|c|c|c|c|c|}
\hline Group & $\begin{array}{c}\text { weak measurement } \\
\text { invariance }\end{array}$ & $\begin{array}{c}\text { strong measurement } \\
\text { invariance }\end{array}$ & $\begin{array}{c}\text { strict measurement } \\
\text { invariance }\end{array}$ & $\begin{array}{c}\text { factor variance } \\
\text { invariance }\end{array}$ & $\begin{array}{c}\text { factor covariance } \\
\text { invariance }\end{array}$ \\
\hline gender & invariance & non-invariance & non-invariance & invariance & invariance \\
\hline grade7-grade 8 & non-invariance & invariance & non-invariance & invariance & invariance \\
\hline grade8-grade 9 & invariance & invariance & non-invariance & non-invariance & non-invariance \\
\hline grade7-grade 9 & invariance & invariance & non-invariance & invariance & invariance \\
\hline
\end{tabular}

As a result, factor loading and item intercept is generally same pattern except some scalar is different factors across gender. Thirdly, test error variance invariance showed that are non-invariance for all. However, rejecting the hypothesis of strict measurement invariance does not mean RSI are problematic but only implies that the amount of variances of the observed item responses that are unexplained by their underlying factors are non-invariance across group (Wang \& Wang, 2012). Therefore RSI is a measurement invariance across Grade 8-Grade 9 and across Grade 7-Grade 9. In contrast, across gender and across Grade 7-Grade 8 is non-invariance of measurement invariance. However, the empirical data are confirmed with theory of research skills. In other word, RSI does the most construct validity.

The structural invariance is testing for structural equivalence, focus on the factor covariance. Some researchers may also wish to test for the equality of the factor variance (Byrne, 2008). However our study is limited to test both factor covariance and factor variance. As conclusion, results revealed that only test invariance across Grade 8 and Grade 9 is non-invariance.

\section{Acknowledgements}

This work was supported by the Higher Education Research Promotion and National Research University Project of Thailand, Office of the Higher Education Commission, through the Cluster of Research to Enhance the Quality of Basic Education.

\section{References}

Asparouhov, T., \& Muthen, B. (2014). Multiple-group factor analysis alignment. Structural Equation Modeling: A Multidisciplinary Journal, 21(4), 495-508. doi: 10.1080/10705511.2014.919210.

Bas, A.U., \& Yurdabakan, I. (2012). Factor structure of the reactive-proactive aggression questionnaire in Turkish children and gender, grade-level, and socioeconomic status differences in reactive and proactive aggression. Journal of Psycho-educational Assessment, 30(3), 284-297. doi: 10.1177/0734282911428892.

Bentler, P.M. (1992). On the fit of models to covariances and methodology to the Bulletin. Psychological Bulletin, 112(3), $400-404$.

Bryne, B.M. (2008). Testing for multigroup equivalence of a measuring instrument: A walk through the process. Comprobando la equivalencia multigrupal de un instrument de medida: pasos del proceso, 20(4), 872-882.

Byrne, B.M. (2012). Structural Equation Modeling with Mplus: Basic Concepts, Applications, and Programming. New York: Routledge.

Canivez, G.L., Neitzel, R., \& Martin, B.E. (2005). Construct validity of the Kaufman Brief Intelligence Test, Wechsler Intelligence Scale for Children - third edition, and Adjustment Scales for Children and Adolescents. Journal of Psycho-educational Assessment, 
23(1), 15-34. doi: $10.1177 / 073428290502300102$.

Canivez, G.L., \& Sproul, K. (2005). Assessing the construct validity of the adjustment scales for children and adolescents. Journal of Psycho-educational Assessment, 23(1), 3-14. doi: 10.1177/073428290502300101.

Czarneski, D. (2013). A First-year course that teachers research skills. PRIMUS, 23(5), 487-497. doi: 10.1080/10511970.2013.764365.

Dimitrov, D.M. (2010). Testing for factorial invariance in the context of construct validation. Measurement \& Evaluation in Counseling \& Development, 43(2), 121-149. doi: 10.1177/0748175610373459.

Elizabeth, V., \& Grant, B.M. (2013). The spirit of research has changed: Reverberations from researcher identities in managerial times. Higher Education Research \& Development, 32(1), 122-135. doi: 10.1080/07294360.2012.751362.

Hair, J.F. (2006). Multivariate data analysis. (6 $6^{\text {th }}$ ed.). Upper Saddle River, N.J.: Prentice-Hall International.

Harrell-Williams, L.M., Sorto, M.A., Pierce, R.L., Lesser, L.M., \& Murphy, T.J. (2014). Validation of scores from a new measure of preservice teachers' self-efficacy to teach statistics in the middle grades. Journal of Psycho-educational Assessment, 32(1), 40-50. doi: $10.1177 / 0734282913486256$.

Hu, L.T., \& Bentler, P.M. (1999). Cutoff criteria for fit indexes in covariance structure analysis: Alternatives. 6(1), Structural Equation Modeling, 1-55. doi: 10.1080/10705519909540118.

Kiley, M., Moyes, T., \& Clayton, P. (2009). To develop research skills: Honors programs for the changing research agenda in Australian universities. Innovations in Education and Teaching International, 46(1), 15-25. doi: 10.1080/14703290802646164.

Leat, D., \& Reid, A. (2012). Exploring the role of student researchers in the process of curriculum development. Curriculum Journal, 23(2), 189-205. doi: 10.1080/09585176.2012.678691.

Lowe, P.A. (2014). Should test anxiety be measured differently for males and females? Examination of measurement bias across gender on measures of text anxiety for middle and high school, and college students. Journal of Psycho-educational Assessment, 33(3), 238-246. doi: 10.1177/0734282914549428.

MacCallum, R.C., Browne, M.W., \& Sugawara, H.M. (1996). Power analysis and determination of sample size for covariance structure modeling. Psychological Methods, 1(2), 130-149.

Marsh, H.W., Hau, K.T., \& Wen, Z. (2004). In search of golden rules: Comment on hypothesis-testing approaches to setting cutoff values for fit indexes and dangers in over generalizing Hu and Bentler's (1999) findings. Structural Equation Modeling, 11(3), 320-341.

Martin, A.J., \& Marsh, H.W. (2008). Workplace and academic buoyancy psychometric assessment and construct validity amongst school personnel and students. Journal of psycho-educational assessment, 26(2), 168-184. doi: 10.1177/0734282907313767.

Meerah, T.S.M., Osman, K., Zakaria, E., Ikhsan, Z.H., Krish, P., Lian, D.K.C., \& Mahmod, D. (2012). Developing an instrument to measure research skills. Procedia-Social and Behavioral Sciences, 60, 630-636. doi: 10.1016/j.sbspro.2012.09.434.

M.W. Browne, R.C. (1992). Alternative ways of assessing model fit. Sociological methods \& Research, 21(2). doi: 10.1177/004912419 2021002005

Sharma, S., Mukherjee, S., Kumar, A., \& Dillon, W.R. (2005). A simulation study to investigate the use of cutoff values for assessing model fit in covariance structure models. Journal of Business Research, 58, 935-943. doi: 10.1016/j.jbusres.2003.10.007

Siegling, A.B., Furnham, A., \& Petrides, K.V. (2015). Trait emotional intelligence and personality gender-invariant linkages across different measures of the big five. Journal of Psycho-educational assessment, 33(1), 57-67. doi: 10.1177/0734282914550385.

Srikoon, S., Bunterm, T., Samranjai, J., \& Wattanathorn, J. (2014). Research synthesis of research-based learning for education in Thailand. Procedia-Social and Behavioral Sciences, 116, 913-917.

Stappenbelt, B. (2013). The effectiveness of the teaching-research nexus in facilitating student learning. Engneering Education, 8(1), 111-121. doi: 10.11120/ened.2013.00002.

Steiger, J.H. (1990). Structural model evaluation and modification: An interval estimation approach. Multivariate Behavioral Research, 25(2), 173-180. doi: 10.1207/s15327906mbr2502_4.

Stokking*, K., Schaaf, M., Jaspers, J., \& Erkens, G. (2004). Teachers' assessment of students' research skills. British Educational Research Journal, 30(1), 93-116. doi: 10.1080/01411920310001629983.

Wang, J., \& Wang, X. (2012). Structural equation modeling: Applications using Mplus. West Sussex, UK: Higher Education Press.

Wannapiroon, P. (2014). Development of research-based blended learning model to enhance graduate students' research competency and critical thinking skills. Procedia-Social and Behavioral Sciences, 136, 486-490.

Weis, R., \& Smenner, L. (2007). Construct validity of the behavior assessment system for children (BASC) self-report of personality evidence from adolescent referred to residential treatment. Journal of Psycho-educational Assessment, 25(2), 111-126. doi: 10.1177/0734282906293762.

Wilkin, C.L. (2014). Enhancing the AIS curriculum: Integration of a research-led, problem-based learning task. Journal of Accounting Education, 32(2), 185-199. doi: 10.1016/j.jaccedu.20.14.04.001.

Willison, J.O.K. (2007). Commonly know, commonly not know, totally unknown: A framework for students becoming researchers. The Higher Education Research and Development, 26(4), 393-409.

Zehra, N., Hassaan, A., \& Mushtaq, S. (2015). Research amongst junior and senior medical students: Comparison of knowledge, attitude and practice. Professional Medical Journal, 22(1), 121-126. 